\title{
In vitro characterization of the percutaneous absorption of tramadol into inner ear domestic feline skin using the Franz skin finite dose model
}

August S. Bassani ${ }^{\dagger}$, Daniel Banov ${ }^{\dagger}$, Chris Simmons $^{\dagger}$ and Ha Phan ${ }^{*}$

*Correspondence: hphan@pccarx.com

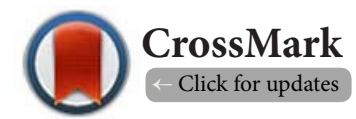

${ }^{\dagger}$ These authors contributed equally to this work.

Professional Compounding Centers of America (PCCA), Houston, Texas, USA.

\begin{abstract}
Pain, whether it is acute or chronic, can induce stress and reduce overall quality of life for domestic felines. Tramadol, a synthetic opioid that exerts analgesic effects by binding to $\mu$-opioid receptors as well as inhibiting neuronal reuptake of norepinephrine and serotonin, can be used alone or in combination with NSAIDs for the treatment of domestic feline pain. Tramadol can be incorporated into transdermal bases such as PLO or Lipoderm for transdermal delivery. The purpose of this study is to characterize the percutaneous absorption of two tramadol formulations (Tramadol $100 \mathrm{mg} / \mathrm{g}$ in PLO and Tramadol 100 $\mathrm{mg} / \mathrm{g}$ in Lipoderm), when applied to the inner ear of domestic feline skin, in vitro, using the Franz skin finite dose model. A variable finite dose (e.g., $25 \mu \mathrm{g} / \mathrm{cm}^{2}$ ) of each formulation was applied to skin sections from two donors and cultured within Franz diffusion cells for $48 \mathrm{hr}$. Tramadol total absorption, rate of absorption, surface wash, and skin content were quantified using HPLC analysis. Results show that both PLO and Lipoderm were capable of facilitating the percutaneous absorption of tramadol across ex-vivo domestic feline inner ear skin. However, tramadol content within surface wash $(3.65 \% \pm 0.14)$ was lower for the Lipoderm formulation, which potentially correlates to the higher total absorption (100.40\% \pm 3.24$)$ of tramadol when in Lipoderm. Although the rise to peak rate of absorption was approximately $2.5 \mathrm{hr}$ for both formulations, the decline in rate was steadier and more predictable for tramadol in Lipoderm than in PLO. Results of this study can help practitioners and pharmacists predict the in vivo percutaneous absorption of tramadol in domestic felines. The prediction can then guide them in selecting the most favorable transdermal base when prescribing and compounding with tramadol for domestic feline use.
\end{abstract}

Keywords: Domestic feline(s), pain, pharmaceutical compounding, percutaneous absorption, tramadol, Lipoderm, PLO, Franz skin finite dose model

\section{Introduction}

Companion animals such as cats and dogs are popular pets in many countries around the world. In the United States, the estimated percentages of households owning pets are $30.4 \%$ for cats and $36.5 \%$ for dogs [1]. Though both domestic animals are cherished by their caregivers, cats are often under-treated for pain in comparison to dogs. Domestic cats, also referred to as domestic felines, belong to the larger family of felines, which also includes tigers, lions, jaguars, and wild cats. Only $56 \%$ of domestic felines receive adequate analgesia due to the increased difficulty for caregivers to recognize and assess pain in these animals [2].

The types of pain experienced by domestic felines can be divided into two categories: acute pain and chronic pain. Acute pain is often a result of trauma or surgery, while chronic pain is usually associated with Degenerative Joint Disease (DJD), a condition that involves deterioration of tissues surrounding the joints. Opioids such as morphine or fentanyl are commonly used for acute pain, while Nonsteroidal Anti-Inflammatory Drugs (NSAIDs) such as meloxicam or ketoprofen are commonly used for chronic pain [3]. Though opioids and NSAIDs are common treatment options, there are disadvantages with the use of these medications in domestic felines. As obligated carnivores, the feline family has low capacity for the metabolism of drugs via hepatic glucuronidation, a pathway that develops as a result of exposure to phytoalexins in plants. Deficiency in this pathway can lead to decreased efficacy with morphine, as the glucuronidation pathway is required to convert morphine to 
Bassani et al. Veterinary Medicine and Animal Sciences 2015,

its active metabolite, Morphine-6-Glucuronide (M-6-G) $[2,3]$. Although NSAIDs do not require the glucuronidation pathway for activation, long-term exposure to this medication class can lead to gastrointestinal toxicity (e.g., bleeding, ulcerations) and renal damage, which limits the chronic use of NSAIDs [3]. Due to the limitations of current therapies, pain management in domestic felines has become increasingly challenging for practitioners and pharmacists.

In recent years, tramadol has been researched for the treatment of domestic feline pain. Tramadol is a synthetic opioid that exerts analgesic effects by binding to $\mu$-opioid receptors as well as inhibiting neuronal reuptake of norepinephrine and serotonin [4]. Previous studies have shown that tramadol can be used alone or in combination with NSAIDs for the treatment of mild to moderate pain in domestic felines $[5,6]$. The conventional routes of administration for tramadol include oral, Intravenous (IV), and Subcutaneous (SC) administration [5]. Both IV and SC administration are invasive and, therefore, it may be challenging for caregivers to successfully administer tramadol in a home environment. Although oral administration of tramadol is non-invasive, this route of administration requires physical restraint of the animal by its owner to administer each dose, which can lead to non-compliance and treatment failure [3].

The Food and Drug Administration (FDA) has recognized the need for pharmaceutical compounding in veterinary medicine. Particularly with regards to domestic felines, pharmaceutical compounding can be utilized to overcome the limitations of conventional therapies [7]. Pharmaceutical compounding involves the preparation of customized medications to meet the individual patient needs [8]. When compounded, tramadol can be incorporated into transdermal bases such as Pluronic Lecithin Organogel (PLO) or Lipoderm for transdermal delivery. In comparison to oral, IV, and SC administration, transdermal delivery is a non-invasive route of administration that allows for the percutaneous absorption of drugs. Following application, the medication is intended to be directly absorbed into systemic circulation over a period of time, bypassing first-pass metabolism [9].

PLO is a first generation transdermal base that contains Pluronic F-127, soya lecithin, and IPP (Isopropyl Palmitate)/ IPM (Isopropyl Myristate). PLO is composed of two phases, an aqueous phase (pluronic phase) and an oil phase (lecithin phase), continuously mixed with one another until a homogenous gel is formed. The gel is intended to dissolve both hydrophobic and hydrophilic drugs, with hydrophobic drugs dissolving in the oil phase and hydrophilic drugs dissolving in the aqueous phase [10]. Lipoderm, on the other hand, is a transdermal base that consists of a proprietary liposomal component shown to successfully facilitate the penetration of drugs into and through ex vivo human skin under in vitro conditions [11]. Although transdermal delivery of tramadol in PLO or Lipoderm is advantageous in comparison to conventional routes, there is limited data to support the efficacy of compounded tramadol for transdermal delivery in domestic felines. The purpose of this study is to characterize the percutaneous absorption of two tramadol formulations (Tramadol $100 \mathrm{mg} / \mathrm{g}$ in PLO and Tramadol 100 $\mathrm{mg} / \mathrm{g}$ in Lipoderm), when applied to the inner ear of domestic feline skin, in vitro, using the Franz skin finite dose model. In domestic felines, transdermal delivery usually involves the rubbing of medication to the animal's inner ear skin surface, a location that cannot be licked and is usually hairless [12]. The Franz skin finite dose model has proven to be a valuable tool in predicting in vivo percutaneous absorption of topically applied drugs $[11,13]$. Therefore, this model was selected to characterize the percutaneous absorption of tramadol into and through the ex vivo inner ear skin of domestic feline donors.

\section{Materials and methods \\ Materials}

Details relating to the composition of the samples used for testing are shown in Table 1.

Table 1. Composition of the samples used for testing.

\begin{tabular}{llll}
\hline \multicolumn{4}{c}{ Tramadol 100 mg/g in PLO } \\
\hline Ingredients & Quantity & Lot number & Source \\
\hline $\begin{array}{l}\text { Tramadol HCl } \\
\text { Propylene glycol }\end{array}$ & $8 \mathrm{~g}$ & $\mathrm{~g} 129073$ & PCCA \\
$\begin{array}{l}\text { Lecithin isopropyl } \\
\text { palmitate base }\end{array}$ & $22 \mathrm{~g}$ & $\mathrm{C} 129628$ & PCCA \\
$\begin{array}{l}\text { Poloxamer } 407 \text { 20\% } \\
\text { gel }\end{array}$ & q.s. to $100 \mathrm{~g}$ & 1722672 & PCCA \\
\hline \multicolumn{4}{c}{ Tramadol $\mathbf{1 0 0} \mathbf{~ m g} / \mathbf{g}$ in Lipoderm } \\
\hline $\begin{array}{l}\text { Tramadol HCl } \\
10 \mathrm{~g}\end{array}$ & C129073 & PCCA \\
$\begin{array}{l}\text { Propylene glycol } \\
\text { Lipoderm }\end{array}$ & $8 \mathrm{~g}$ & C129628 & PCCA \\
\hline
\end{tabular}

\section{Tramadol $100 \mathrm{mg} / \mathrm{g}$ in PLO}

Tramadol hydrochloride $(\mathrm{HCl})$, in an amount necessary to result in a concentration of $100 \mathrm{mg} / \mathrm{g}$, was added to Lecithin Isopropyl Palmitate solution base along with $10 \%$ Propylene Glycol as a wetting agent. Poloxamer $40720 \%$ gel was then added in sufficient quantity to reach the final weight. The formulation was mixed using an electronic mortar and pestle (EMP) for 3 min on a setting of 7 , sheared twice using an ointment mill at a setting of 1 , and remixed for $1 \mathrm{~min}$ on a setting of 5 . The specific gravity of the final formulation was $1.002 \mathrm{~g} / \mathrm{mL}$.

\section{Tramadol $100 \mathrm{mg} / \mathrm{g}$ in Lipoderm}

Tramadol $\mathrm{HCl}$, in an amount necessary to result in a concentration of $100 \mathrm{mg} / \mathrm{g}$, was added to Lipoderm along with $10 \%$ Propylene Glycol as a wetting agent. The formulation was mixed with the aid of an EMP for 3 min at a setting of 7, sheared twice using an ointment mill on a setting of 1 , and remixed with an 
EMP for $1 \mathrm{~min}$ at a setting of 5 to achieve accurate content uniformity. The density of the final formulation was $0.726 \mathrm{~g} /$ $\mathrm{mL}$, thus the final tramadol $\mathrm{HCl}$ concentration in weight per volume was $72.6 \mathrm{mg} / \mathrm{mL}$.

\section{Skin preparation}

The percutaneous absorption of two tramadol formulations were evaluated using normal feline ventral inner ear skin obtained from two different donors, a domestic short hair feline female (donor 1) and male (donor 2). Prior to use, the skin was thawed in water at approximately $37^{\circ} \mathrm{C}$ and rinsed with tap water to remove blood and other material from the surface. The ventral (inner) surface of the skin was then separated from the dorsal layer. The skin from the two donors were cut into multiple sections to fit nominal $0.8 \mathrm{~cm}^{2}$ Franz diffusion cells, a chamber that cultures the skin cells and allows for the condition of the skin to be maintained at a temperature and humidity that matches in vivo conditions [13]. Seven skin cross-sections were obtained from the two donors. Each skin section was assigned to one chamber, with four chambers for donor 1 and three for donor 2 (Table 2). Within the chambers, the cells were mounted on a diffusion apparatus so that the epidermal surface is exposed to the laboratory environment via the chimney (Figure 1). The receptor compartment of the chamber was filled to capacity with isotonic PhosphateBuffered Saline (PBS), pH 7.4 \pm 0.1 , stirred magnetically at approximately $600 \mathrm{rpm}$. The skin surface was maintained at a temperature of $32^{\circ} \mathrm{C} \pm 1^{\circ} \mathrm{C}$.

\section{Dosing and sample collection}

Prior to dosing, the receptor solution was removed and replaced with fresh solution containing $0.1 \times P B S$ with $0.1 \%$

Table 2. Dosing details for the seven chambers.

\begin{tabular}{lccccccc}
\hline & \multicolumn{3}{c}{ Donor 1 } & \multicolumn{3}{c}{ Donor 2 } \\
\hline Skin chamber & 1 & 2 & 3 & 4 & 5 & 6 & 7 \\
Tramadol in PLO & X & X & -- & -- & X & -- & -- \\
Tramadol in Lipoderm & -- & -- & X & X & -- & X & X \\
\hline
\end{tabular}

Volpo. The chimney was next removed to allow for access to the epidermal surface for dose application. The two formulations were tested on duplicate sections from two different donors for percutaneous absorption of tramadol over a $48 \mathrm{hr}$ dosing period. Due to the odd number of skin sections available from the two donors, tramadol in PLO was applied to three skin sections (two from donor 1 and one from donor 2) while tramadol in Lipoderm was applied to four skin sections (two from each donor) (Table 2). Using the finite dose technique, a variable finite dose (e.g., $25 \mu \mathrm{g} /$ $\mathrm{cm}^{2}$ ) of each formulation was applied to the outer surface of each skin section using a positive displacement pipette set to deliver $5 \mu \mathrm{L}$ formulation $/ \mathrm{cm}^{2}$ [11]. The dose was then

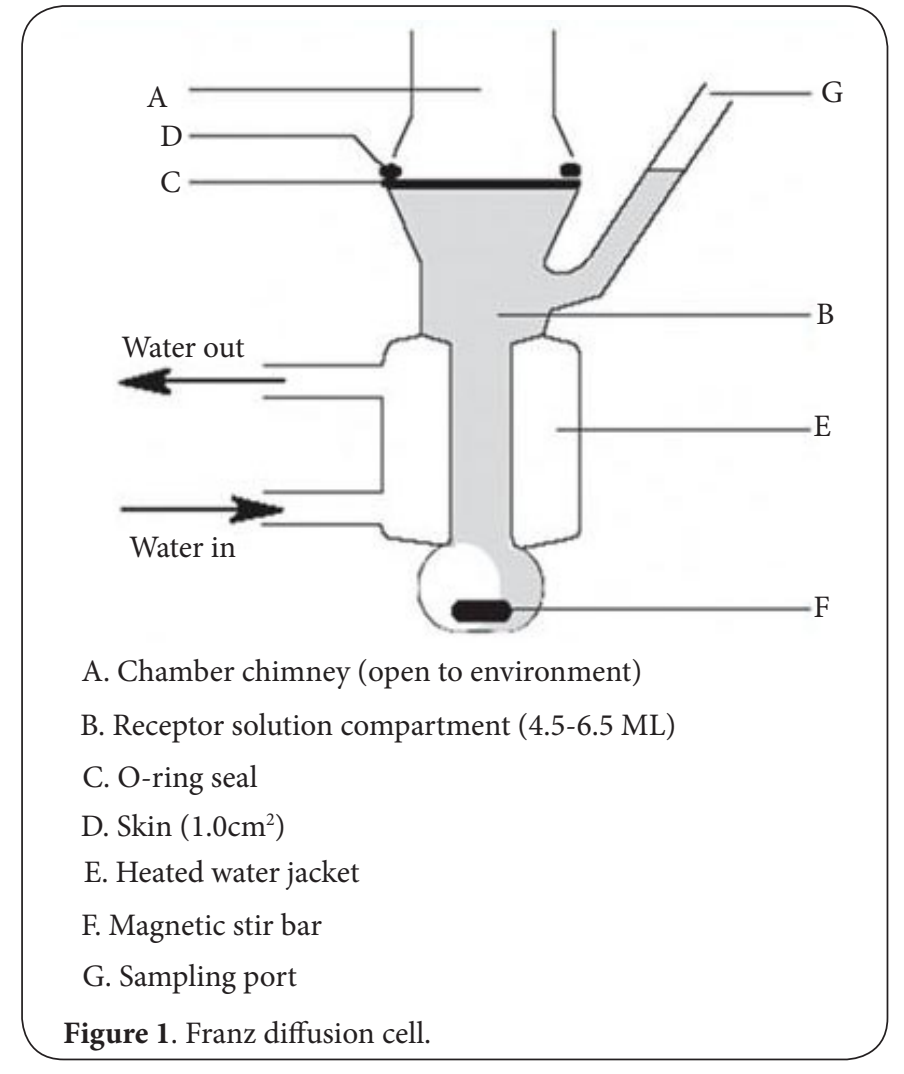

spread across the surface with a glass rod and the chimney was replaced five to ten minutes after dose application. At $2,4,8,12,24,32$, and $48 \mathrm{hr}$ following dose application, the receptor solution was removed, replaced with fresh receptor solution, and a predetermined volume of aliquot was saved for subsequent High-Performance Liquid Chromatography (HPLC) analysis. Samples that were not assayed on the day of collection were stored at or below $-20^{\circ} \mathrm{C}$.

Following collection of the last receptor solution sample at $48 \mathrm{hr}$, surfaces of the skin were washed twice ( $0.5 \mathrm{~mL}$ each time) with 80:20 Ethanol:Water to remove unabsorbed formulation from skin surfaces. The intact skin was then removed from the chamber and extracted in 80:20 Ethanol:Water overnight at room temperature. Tramadol skin content was determined via HPLC analysis of the extractant sample.

\section{Analytical testing}

Tramadol contents within the skin, in the surface wash, and in each receptor solution sample collected at the predetermined time intervals were quantified using HPLC/UV (Ultraviolet). HPLC was conducted on a Hewlett-Packard 1100 Series HPLC system with a diode array detector at a wavelength of 220 $\eta \mathrm{m}(4 \mathrm{\eta m})$ to $500 \mathrm{\eta m}(50 \mathrm{\eta m})$. A solvent system consisting of $70 \%$ Water ( $\mathrm{pH} 9.5$ ) with $10 \mathrm{mM}$ Ammonium Formate and $30 \%$ Methanol was run through a Phenomenex Gemini C18 column $(3 \mu, 50 \times 3 \mathrm{~mm})$ at a flow rate of $0.4 \mathrm{~mL} / \mathrm{min}$. The column temperature was maintained at $25^{\circ} \mathrm{C}$. 
Bassani et al. Veterinary Medicine and Animal Sciences 2015,

\section{Results}

To characterize the percutaneous absorption of tramadol, a total of 4 parameters were determined for each of the 7 chambers, as follows:total absorption, rate of absorption, surface wash, and skin content. Since each chamber had corresponding values for the 4 parameters, mean values and standard deviation were calculated for each parameter across the 2 donors. Mean values were expressed as percentages of the applied dose to further compare the abilities of PLO and Lipoderm in facilitating the percutaneous absorption of tramadol. Summary of res-ults across the two donors can be found in Tables $\mathbf{3}$ and $\mathbf{4}$ and Figure 2.

Total absorption $(\mu \mathrm{g})$ was calculated as the sum of tramadol content $(\mu \mathrm{g})$ within the 7 samples collected over $48 \mathrm{hr}$. Mean total absorption $(\mu \mathrm{g})$ was the average of total absorptions for all chambers from the 2 donors. The mean total absorption of tramadol in PLO was $333.70 \mu \mathrm{g} \pm 43.09$, corresponding to $90.28 \% \pm 14.70$ absorption of the applied dose. For tramadol in Lipoderm, mean total absorption was $277.10 \mu \mathrm{g} \pm 11.39$, corresponding to $100.40 \% \pm 3.24$ absorption of the applied dose.

Rate of absorption, presented as flux ( $\mu \mathrm{g} / \mathrm{cm}^{2} / \mathrm{hr}$ ) of tramadol into receptor solution, was determined by dividing the amount of tramadol absorbed during a time interval and the length of that interval. Flux is a value reported at midpoint of sample collection for a sampling period. For instance, if samples are collected at 4 and $8 \mathrm{hr}$, the flux of tramadol during that time interval is equal to the amount of tramadol in the receptor solution between 4 and $8 \mathrm{hr}$, divided by the time span of $4 \mathrm{hr}$. Flux is then plotted at $6 \mathrm{hr}$, midpoint of 4 and $8 \mathrm{hr}$. Mean flux $\left(\mu \mathrm{g} / \mathrm{cm}^{2} / \mathrm{hr}\right)$, shown in Table 4, was calculated across donors

Table 3. Across donor summary: mean total absorption, surface wash and skin content (mean \pm SE (standard error) as percent applied dose, $n=2$ ).

\begin{tabular}{lllllll}
\hline & $\begin{array}{l}\text { Total } \\
\text { absorption }(\boldsymbol{\mu g})\end{array}$ & $\begin{array}{l}\text { Total } \\
\text { absorption }(\%)\end{array}$ & $\begin{array}{l}\text { Surface } \\
\text { wash }(\boldsymbol{\mu g})\end{array}$ & $\begin{array}{l}\text { Surface } \\
\text { wash }(\%)\end{array}$ & $\begin{array}{l}\text { Skin } \\
\text { content }(\boldsymbol{\mu g})\end{array}$ & $\begin{array}{l}\text { Skin } \\
\text { content }(\%)\end{array}$ \\
\hline Tramadol in Lipoderm & $277.10 \pm 11.39$ & $100.40 \pm 3.24$ & $9.86 \pm 0.15$ & $3.65 \pm 0.14$ & $2.97 \pm 0.28$ & $1.09 \pm 0.07$ \\
Tramadol in PLO & $333.70 \pm 43.09$ & $90.28 \pm 14.70$ & $39.66 \pm 54.79$ & $10.45 \pm 14.42$ & $<$ LOD & $<$ LOD \\
\hline
\end{tabular}

Table 4. Across donor summary: mean flux $\left(\mu \mathrm{g} / \mathrm{cm}^{2} / \mathrm{hr}\right)$ (mean \pm SE, $n=2$ donors).

\begin{tabular}{lll}
\hline Time (hr) & Tramadol in PLO & Tramadol in Lipoderm \\
\hline 1 & $17.91 \pm 19.00$ & $13.57 \pm 3.19$ \\
3 & $15.40 \pm 16.28$ & $15.40 \pm 0.71$ \\
6 & $10.32 \pm 8.70$ & $13.80 \pm 0.54$ \\
10 & $10.31 \pm 6.68$ & $14.21 \pm 1.73$ \\
18 & $12.55 \pm 2.78$ & $9.80 \pm 1.02$ \\
28 & $6.48 \pm 3.93$ & $4.36 \pm 1.05$ \\
40 & $4.09 \pm 5.01$ & $1.50 \pm 0.21$ \\
\hline
\end{tabular}

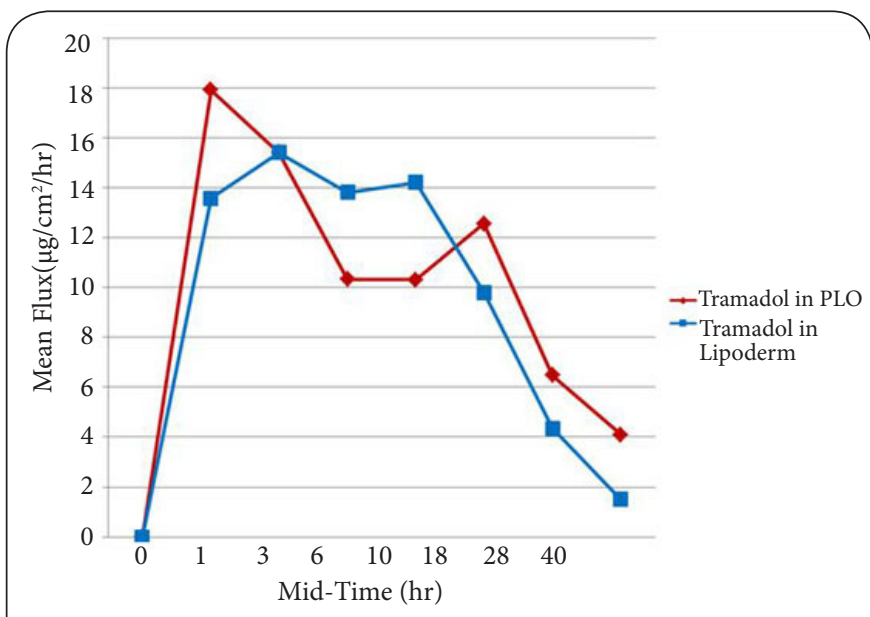

Figure 2. Across donor summary: mean flux $\left(\mu \mathrm{g} / \mathrm{cm}^{2} / \mathrm{hr}\right)$ for two compounded transdermal formulations. for each sampling interval. Figure 2 illustrates mean flux data plotted as the amount of tramadol absorbed through the skin against time.

Surface wash $(\mu \mathrm{g})$ refers to the amount of tramadol remaining on the surface of the skin after $48 \mathrm{hr}$ of dose application. Surface wash was $39.66 \mu \mathrm{g} \pm 54.79$ for tramadol in PLO and 9.86 $\mu \mathrm{g} \pm 0.15$ for tramadol in Lipoderm, corresponding to $10.45 \%$ \pm 14.42 and $3.65 \% \pm 0.14$ of the applied dose, respectively.

Skin content $(\mu \mathrm{g})$ refers to the amount of tramadol found within the skin after $48 \mathrm{hr}$. Mean skin content was calculated as the average tramadol content in the skin sections across 2 donors. Mean tramadol skin content was less than the Lower Limit of Detection (LOD) for tramadol in PLO. For tramadol in Lipoderm, mean skin content was $2.97 \mu \mathrm{g} \pm 0.28$, which is equivalent to a mean of $1.09 \% \pm 0.07$ of the applied dose.

\section{Discussion}

Results of this study show that both PLO and Lipoderm were capable of facilitating the percutaneous absorption of tramadol across ex-vivo feline inner ear skin using the Franz skin finite dose model. For both transdermal formulations (tramadol in PLO and tramadol in Lipoderm), the majority of the applied dose penetrated the skin as more than $90 \%$ was found in the receptor solution after $48 \mathrm{hr}$. However, the mean total absorption of tramadol when in Lipoderm $(100.40 \% \pm 3.24)$ was higher than when in PLO (90.28\% \pm 14.70$)$. The higher mean percent total absorption of tramadol seen with Lipoderm potentially indicates that this transdermal base has greater penetration potentials in comparison to PLO. If the tramadol in PLO and tramadol in Lipoderm are both applied to domestic feline inner 
ear skin, tramadol plasma concentrations may be higher in domestic felines receiving the Lipoderm formulation.

When examining rate of absorption, mean flux profile reveal similar time course of tramadol absorption for PLO and Lipoderm, with a rapid rise to a peak flux within $2.5 \mathrm{hr}$ of dose application for both transdermal formulations. Though tramadol, when in Lipoderm, had a lower peak flux, the decline in rate was more steady, forming a more predictable and reliable absorption profile in comparison to the rapid decline and fluctuations in rate for tramadol in PLO (Figure 2). The steadier decline in flux seen with tramadol in Lipoderm may be helpful when managing domestic feline pain as the analgesic effects of tramadol can slowly taper off overtime rather than having a rapid reduction followed by erratic fluctuations of analgesic effects with PLO.

As for surface wash, tramadol content in the surface wash was lower when in Lipoderm than when in PLO. This difference indicates that more tramadol was absorbed when in Lipoderm. Such results then show that Lipoderm may be more efficient at facilitating the percutaneous absorption of tramadol in comparison to PLO as less tramadol remained on the surface following application of the tramadol in Lipoderm formulation. Surface wash results potentially correlate to the higher total absorption of tramadol seen with Lipoderm.

While very minute amounts of the applied dose were found within the skin, tramadol content in the skin was higher for the Lipoderm than the PLO formulation. The higher tramadol skin content may be due to the ability of Lipoderm to partition into various layers of the skin to a greater extent than PLO. Having higher skin content potentially correlates to a slow and steadier decline in flux as tramadol is slowly released from the skin into receptor solution overtime.

The higher mean total absorption, steadier decline in flux, lower surface wash, and higher skin content seen with the tramadol in Lipoderm formulation show that Lipoderm may be a more appropriate transdermal base for the transdermal delivery of tramadol in comparison to PLO. However, since the study was conducted in vitro, future studies need to be conducted in vivo to fully inform practitioners and pharmacists on the efficacy of tramadol in Lipoderm compared to tramadol in PLO for domestic feline pain.

\section{Conclusions}

Knowledge of this study's results can be used to predict the in vivo rate and extent of percutaneous absorption of tramadol in domestic felines. The data collected can augment the limited efficacy data relating to compounded tramadol for transdermal delivery in domestic felines. Based on the in vitro results of this study, one may likely hypothesize that tramadol, when in Lipoderm, may potentially have a higher total absorption and a steadier decline in rate of absorption in comparison to when in PLO. The prediction can guide practitioners and pharmacists, when prescribing and compounding with tramadol for feline use, to select a transdermal base with the best and most predictable percutaneous absorption potential.

List of abbreviations

DJD: Degenerative Joint Disease

EMP: Electronic Mortar and Pestle

FDA: Food and Drug Administration

HPLC: High-Performance Liquid Chromatography

IPM: Isopropyl Myristate

IPP: Isopropyl Palmitate

IV: Intravenous

LOD: Lower Limit of Detection

NSAIDs: Nonsteroidal Anti-Inflammatory Drugs

PBS: Phosphate-Buffered Saline

PLO: Pluronic Lecithin Organogel

SC: Subcutaneous

UV: Ultraviolet

Competing interests

The authors declare that they have no competing interests.

Authors' contributions

\begin{tabular}{|l|c|c|c|c|}
\hline Authors' contributions & ASB & DB & CS & HP \\
\hline Research concept and design & $\checkmark$ & $\checkmark$ & -- & -- \\
\hline Data analysis and interpretation & $\checkmark$ & $\checkmark$ & -- & $\checkmark$ \\
\hline Writing the article & $\checkmark$ & $\checkmark$ & -- & $\checkmark$ \\
\hline Critical revision of the article & $\checkmark$ & $\checkmark$ & $\checkmark$ & $\checkmark$ \\
\hline Final approval of article & $\checkmark$ & $\checkmark$ & $\checkmark$ & $\checkmark$ \\
\hline
\end{tabular}

\section{Acknowledgement}

The authors would like to thank Paul A. Lehman, MSc (Cetero Research, Fargo, ND, USA) for conducting the Franz finite dose model experiments, which were funded by PCCA.

\section{Publication history}

Editor: Scott Whisnant, North Carolina State University, USA. EIC: Olivier A. E. Sparagano, Coventry University, UK.

Received: 03-Apr-2015 Final Revised: 12-May-2015

Accepted: 19-May-2015 Published: 25-May-2015

\section{References}

1. American Veterinary Medical Association. U.S. Pet Ownership Statistics 2012. 2015. | Website

2. Robertson SA. Assessment and management of acute pain in cats. $J$ Vet Emerg Crit Care. 2005; 15:261-72. | Article

3. Robertson SA. Chapter 24: cat-specific considerations. In J. Gaynor \& W. Muir III (Eds.), Handbook of Veterinary Pain Management. St. Louis, Missouri: Mosby. 2015; 493-516.

4. Takasuga S, Yamamoto R, Mafune S, Sutoh C, Kominami K, Yoshida Y, Ito $\mathrm{M}$ and Kinoshita $\mathrm{M}$. In-vitro and in-vivo transdermal iontophoretic delivery of tramadol, a centrally acting analgesic. J Pharm Pharmacol. 2011; 63:1437-45. | Article | PubMed

5. Ray J, Jordan D, Pinelli C, Fackler B, Boggess D and Clark J. Case studies of compounded Tramadol use in cats. Int J Pharm Compd. 2012; 16:44-9. I Article | PubMed

6. Gaynor J and Muir III W. Chapter 14: Alternative drugs and novel therapies used to treat pain. In J. Gaynor \& W. Muir III (Eds.), Handbook of Veterinary Pain Management. St. Louis, Missouri: Mosby. 2015; 283284.

7. U.S. Food and Drug Administration. Animal drugs. 2015. | Website

8. U.S. Pharmacopeial Convention. Pharmaceutical CompoundingNonsterile Preparations, in: U.S.P. Convention (Ed.) 795, U.S. Pharmacopeial Convention, US, 2014. | Pdf

9. Muir III W. Chapter 15: Factors influencing analgesic drug selection, dose, and routes of drug administration. In J. Gaynor \& W. Muir III (Eds.), 
Bassani et al. Veterinary Medicine and Animal Sciences 2015, http://www.hoajonline.com/journals/pdf/2054-3425-3-3.pdf

Handbook of Veterinary Pain Management. St. Louis, Missouri: Mosby. 2015; 329-330.

10. Belgamwar V, Chauk D, Pandey M and Surana S. Pluronic lecithin organogel. Asian Journal of Pharmaceutics. 2008; 2:134. | Article

11. Bassani AS, Banov D and Lehman PA. Evaluation of the Percutaneous Absorption of Promethazine Hydrochloride, In Vitro, Using the Human Ex Vivo Skin Model. Int J Pharm Compd. 2008; 12:270-73. | Article | PubMed

12. Papich MG. Drug compounding for veterinary patients. AAPS J. 2005 7:E281-7. | Article | PubMed Abstract | PubMed Full Text

13. Franz TJ, Lehman PA and Raney SG. Use of excised human skin to assess the bioequivalence of topical products. Skin Pharmacol Physiol. 2009; 22:276-86. | Article | PubMed Abstract | PubMed Full Text

\section{Citation:}

Bassani AS, Banov D, Simmons $\mathrm{C}$ and $\mathrm{Phan} \mathrm{H}$. In vitro characterization of the percutaneous absorption of tramadol into inner ear domestic feline skin using the Franz skin finite dose model. Vet Med Anim Sci. 2015; 3:3. http://dx.doi.org/10.7243/2054-3425-3-3 